WALKING AGAINST GANGER: THE IMPAGT OF LONGTERM EXERGISE TRAINING ON MAMMARY TUMOURS A.I. Faustino-Rocha ${ }^{*}$, P.A. Oliveira ${ }^{\dagger}$, A. Gama ${ }^{\dagger}$, H. Vala ${ }^{\dagger,}$, F. Esteves , R. Ferreira ${ }^{\$}$ and M. Ginja

* Faculty of Veterinary Medicine, Lusophone University of Humanities and Technologies, Lisbon, ${ }^{\dagger}$ CIT AB, Department of Veterinary Sciences, University of Trás-os-Montes and Alto Douro, Vila Real, ${ }^{\ddagger}$ CI DETS, ESAV, IPV and ${ }^{\S}$ Department of Chemistry, University of Aveiro, Aveiro, Portugal

Introduction: Breast cancer is one of the most frequent cancers among women worldwide. The present work evaluated the effects of long-term exercise training on the development of chemicallyinduced mammary cancer.

Materials and Methods: Procedures followed the European legislation and were approved by the Portuguese Ethics Committee (DGAV, approval number 008961). Thirty 7-week-old female Sprague-Dawley rats were injected intraperitoneally with the carcinogen N-methyl-Nnitrosourea $(\mathrm{MNU})(50 \mathrm{mg} / \mathrm{kg})$. After this, they were randomly divided into two experimental groups: sedentary $(n=15)$ and exercised $(n=15)$. Exercised animals were trained on a treadmill for 35 weeks. At the end of the study, the mammary tumours were collected, fixed in formalin and evaluated histologically. The expression of oestrogen receptor alpha $(\mathrm{OR} \alpha)$ was assessed by immunohistochemistry.

Results: An incidence of $100 \%$ was observed. A total of 51 mammary tumours developed; 28 in the sedentary group and 23 in the exercised animals $(P>0.05)$. Each mammary tumour exhibited more than one histological pattern. A total of 121 mammary lesions was counted; 71 in the sedentary group and 50 in the exercised group $(P=0.056)$. The number of malignant lesions was higher in the sedentary group when compared with the exercised one (39 versus 21) $(P=0.020)$. All mammary lesions exhibited nuclear immunolabelling for OR $\alpha$; the immunoexpression was higher in the exercised group when compared with the sedentary one $(P<0.05)$.

Conclusions: Long-term exercise training reduced the number and malignancy of mammary tumours. Additionally, it increased the $\mathrm{OR} \alpha$ immunoexpression that is an indicator of tumour differentiation and better response to hormone therapy.

\section{NEW THERAPEUTIC APPROACHES TO GHRONIC KIDNEY DISEASE}

A. Nogueira ${ }^{*}$, H. Vala ${ }^{\dagger, \delta}$, C. Nóbrega ${ }^{\dagger}$, C.A. Pires ${ }^{\ddagger}$, B. Colaço ${ }^{\ddagger}$, , P.A. Oliveira ${ }^{\ddagger, \S}$ and M.J. Pires

${ }^{*} I P B$, Braganca,${ }^{\dagger}$ CI DET, ESAV, IPV,${ }^{\ddagger} U T A D$ and ${ }^{\S} C I T A B, U T A D$, Portugal

Introduction: The main research goal in patients with chronic kidney disease (CKD) is the development of new therapeutic approaches capable of slowing down the progression to end-stage renal disease. The aim of this work was to evaluate the effects of long-term administration of chaetomellic acid A (CAA), which selectively blocks $\mathrm{H}$ Ras farnesylation, on chronic kidney lesions in $5 / 6$ nephrectomized Wistar rats, an animal model of chronic renal disease.

Materials and Methods: Sixty male Wistar rats were sham-operated (SO) or submitted to 5/6 nephrectomy (RMR). One week after surgery, surviving animals were distributed into four groups: $\mathrm{SO}-\mathrm{SO}$ rats receiving no treatment $(n=13) ; \mathrm{SO}+\mathrm{CAA}-\mathrm{SO}$ rats receiving CAA treatment $(n=13)$; RMR-RMR rats receiving no treatment $(n=14) ; \mathrm{RMR}+\mathrm{CAA}-\mathrm{RMR}$ rats receiving CAA treatment $(n=13)$. CAA was administered intraperitoneally three times a week for 6 months. Renal fibrosis was evaluated by ultrasonography and histopathological analysis. All experimental procedures followed the European (European Directive 2010/63/EU) and National (Decree-Law 113/2013) legislation on the protection of the animals used for scientific purposes.

Results: The kidneys of the RMR animals treated with CAA showed a significant decrease in medullary echogenicity $(P<0.05)$ compared with the RMR rats that received no treatment. Glomerulosclerosis and arteriolosclerosis scores were significantly lower $(P<0.001)$ in the RMR + CAA group when compared with the RMR group.

Gonclusion: These data suggest that pharmacological inhibition of H-Ras proteins activation may be a future strategy in the prevention of end-stage renal disease.

\section{GHRONIC MILD STRESS INCREASES DENTIN HYPERSENSITIVITY AND INDUGES ASTROGLIOSIS IN THE PREFRONTAL GORTEX AND HYPOTHALAMUS IN RATS}

F.M. Barbosa *, D. Cabral ${ }^{\dagger}$, F. Kabadayan ${ }^{*}$, E.F. Bondan ${ }^{\dagger}$, M.F.M. Martins ${ }^{\dagger}$, T.B. Kirsten ${ }^{\dagger}$, L.V. Bonamin ${ }^{\dagger}$, N. QueirozHazarbassanov , M.M. Bernardi ${ }^{*, \dagger}$ and C.H.C. Saraceni ${ }^{*}$

* Graduate Program in Dentistry, ${ }^{\dagger}$ Department of Environmental and Experimental Pathology, University Paulista and ${ }^{\ddagger}$ Department of Pathology, School of Veterinary Medicine, University of São Paulo, Brazil

Introduction: Although depression and pain are common comorbidities, their interaction is not fully understood. This study evaluated the nociceptive response induced by dentin hypersensitivity after dental erosion in rats that were submitted to unpredictable chronic mild stress (UCMS)-induced depressive-like behaviour.

Materials and Methods: Adult male rats were subjected to UCMS (depression [D] group) or not (no depression [ND] group) for 30 days and received either acidic solution to induce dental erosion $(\mathrm{E})$ or water $(\mathrm{W})$, thus forming the WND, END, WD and ED groups. After the end of treatment, depressive-like parameters (i.e. sucrose preference and immobility in the forced swim test) and dentin hypersensitivity were evaluated. Plasma tumour necrosis factor (TNF)- $\alpha$ and corticosterone levels were measured, and astrocytic glial fibrillary acidic protein (GFAP) expression was evaluated in the prefrontal cortex, hippocampus, amygdala and hypothalamus.

Results: Administration of the acidic solution potentiated dentin hypersensitivity and increased corticosterone levels in the ED group compared with the WD group. TNF- $\alpha$ levels only increased in the ED group. The ED group exhibited an increase in astrocytic GFAP expression in the hypothalamus and prefrontal cortex, but decreased in the hippocampus.

Conclusions: These results suggest that UCMS exacerbated the nociceptive response associated with dentin hypersensitivity, concomitant with an increase in plasma corticosterone levels. Hypothalamic and prefrontal cortex astrogliosis in the ED group may be attributable to the increase in corticosterone associated to the UCMS procedure. The reduction of astrocytic GFAP expression in the hippocampus in the ED group supports the association between depression and dentin hypersensitivity. 